Mini review

\title{
Role of osteopontin in dendritic cell shaping of immune responses
}

\author{
Annalisa Del Prete ${ }^{\mathrm{a}, \mathrm{b}}$, Sara Scutera ${ }^{\mathrm{c}}$, Silvano Sozzani ${ }^{\mathrm{a}, *, 1}$, Tiziana Musso ${ }^{\mathrm{c}, 1}$ \\ ${ }^{a}$ Department of Molecular and Translational Medicine, University of Brescia, Brescia, Italy \\ ${ }^{\mathrm{b}}$ Humanitas Clinical and Research Center-IRCCS Rozzano-Milano, Italy \\ ${ }^{\mathrm{c}}$ Microbiology section, Department of Public Health and Pediatric Sciences, University of Torino, Turin, Italy
}

\section{A R T I C L E I N F O}

\section{Keywords:}

Osteopontin

Dendritic cells

$\mathrm{T}$ cell polarization

Autoimmune diseases

Cancer

Mesenchymal stromal cells

\begin{abstract}
A B S T R A C T
Osteopontin (OPN) is a pleiotropic cytokine produced both by immune and non-immune cells and active on different cellular targets. OPN production has been associated with several pathological conditions, including autoimmune diseases (e.g. lupus, multiple sclerosis and rheumatoid arthritis) and cancer. Emerging evidence suggests that the role of OPN has been underestimated, as it seems to be working at multiple levels of immune regulation, such as the shaping of $\mathrm{T}$ cell effector responses, the regulation of the tumor microenvironment, and the functional interaction with mesenchymal stromal cells. In this context, dendritic cells (DCs) play a crucial role being both an important source and a cellular target for OPN action. DC family is composed by several cell subsets endowed with specific immune functions. OPN exerts its biological functions through multiple receptors and is produced in different intracellular and secreted forms. OPN production by DC subsets is emerging as a crucial mechanism of regulation in normal and pathological conditions and starts to be exploited as a therapeutic target. This review will focus on the role of DC-derived OPN in shaping immune response and on the complex role of this cytokines in the regulation in immune response.
\end{abstract}

\section{Introduction}

Osteopontin (OPN), is a complex cytokine and adhesion protein that contains an integrin-binding RGD (arginine-glycine-aspartic acid) sequence commonly found in extracellular matrix molecules. Major functional domains of the protein are conserved among species implying important shared activities [1]. OPN was originally identified as a sialoprotein produced by osteoblasts and crucial, as the name implies, in bone homeostasis [2]. Independently, it was also associated with neoplastic transformation as a tumor-secreted phosphoprotein (SSP-1) [3]. A role of secreted OPN (sOPN) in modulating the immune responses was established only a few years later when OPN turned out to be the cytokine produced by activated lymphocytes and macrophages and called early T-lymphocyte activation-1 (Eta-1) [4]. Since the initial discovery, OPN has been shown to be present in various tissues and body fluids (e.g. milk, urine and serum) and to control a wide range of cellular processes such as motility, adhesion and survival [5]. The functional heterogeneity can be explained in part by the OPN ability to interact with multiple receptors, which include integrins $(\alpha v \beta 1, \alpha v \beta 3$, $\alpha v \beta 5, \alpha v \beta 6, \alpha 4 \beta 1, \alpha 5 \beta 1, \alpha 8 \beta 1$, and $\alpha 9 \beta 1)$ and CD44 variants [6,7]. Additionally, the existence of modified isoforms of sOPN as well as of an intracellular form (iOPN) may also account for OPN pleiotropic activities [8].

Physiological roles of OPN include regulation of developmental processes, tissue remodeling, and immune functions. Importantly, aberrant expression of OPN is closely associated with cardiovascular and kidney diseases, cancer, and diabetes [7]. Moreover, the upregulation of OPN expression by immune cells is associated with the exacerbation of chronic inflammatory diseases such as Crohn's disease, atherosclerosis, and autoimmune diseases (including lupus, multiple sclerosis and rheumatoid arthritis) [5,6,9-11].

OPN is produced by several immune cells including macrophages, $\mathrm{T}$ lymphocytes and dendritic cells (DCs) [6]. DCs are a heterogeneous population of cells localized in different tissues and able to discriminate between immunity and tolerance depending on their activation status. A multiplicity of human and mouse DC subsets can be distinguished based on their origins, anatomical locations, surface phenotype, migratory and functional properties [12-14]. Under steady state conditions, DCs generally derive from bone marrow (BM) precursors and are broadly categorized in conventional/classical (cDCs), traditionally recognized as professional antigen presenting cells, and plasmacytoid DCs (pDCs) known mainly for their ability to secrete type I IFN in response to viral infections [15-17]. Under inflammatory conditions and in selective tissues (e.g. lung, skin, intestine) also monocytes can

\footnotetext{
* Corresponding author at: Department of Molecular and Translational Medicine, University of Brescia, Viale Europa 11, 25123, Brescia, Italy.

E-mail address: silvano.sozzani@unibs.it (S. Sozzani).

${ }^{1}$ These authors have contributed equally to this work.
} 
differentiate in the so called monocyte-derived DCs (moDCs/inflammatory DCs). Additionally, Langerhans cells (LCs) represent a distinct epidermal DC subset that possesses embryonic origin and peculiar life cycle [18]. DCs are equipped with various cell surface receptors to recognize microbial and tissue damaged molecular profiles and induce a wide range of $\mathrm{T}$ cell responses, including effector/helper Th1, Th2, Th22 and Th17 polarization and the generation of regulatory $\mathrm{T}$ cells (Treg) [19]. The diverse functions of DCs depend on the heterogeneity of DC subsets, their maturation state and the type of cytokines secreted. In steady state, immature DCs capture self-antigens or apoptotic cells and migrate to secondary lymphoid organs contributing to the maintenance of tolerance. On the other hand, pathogen-primed mature DCs are essential for the activation of effector T cells [19]. Emerging evidence supports the role of tissue microenvironment in regulating the function of DCs. For example, splenic stromal cells drive mature DCs to acquire regulatory functions, while intestinal stromal cells enable DCs to prime Th2 responses through the production of TSLP (thymic stromal lymphopoietin). Mesenchymal stromal cells (MSCs) are also known to modulate DC differentiation and functional properties [20]. Interestingly, the crosstalk between distinct DC subsets such as pDCs and cDCs has been demonstrated to be relevant in shaping immune response [21,22].

Several studies have shown that DCs are both a source and a target of OPN in normal and pathological responses. Early evidence pointed to sOPN as an important player in DC maturation, migration, and polarization [23-25]; however, the interest regarding the interplay between OPN and DCs has grown remarkably with the recognition that both sOPN and iOPN might influence DC subsets recruitment and cytokine production, thus contributing to $\mathrm{T}$ cell polarization [8].

This review will focus on DCs as producers and targets of OPN summarizing recent findings on the regulation of OPN production (Fig. 1) as well as the autocrine/paracrine effects of OPN on DC functions (Table 1). Emphasis will be given to DC-derived OPN in the balance between regulatory and effector $\mathrm{T}$ cell responses and in the pathogenesis of infections, autoimmunity, allergy, and cancer. In addition, this review will highlight recent findings on the role of DCderived OPN on MSC functions and on the biological relevance of DCMSC interplay in steady state and inflammatory conditions.

\section{OPN structure and receptors}

OPN is encoded by a single copy gene located in the SIBLING (small integrin-binding ligand, N-linked glycoproteins) cluster on the human chromosome 4 and on mouse chromosome 5 [26]. Single nucleotide polymorphisms in the OPN gene are associated with development and/ or disease activity of several autoimmune diseases [27]. The gene has 7 exons, 6 of which are translated in the full-length isoform (referred to as $\mathrm{OPN}-\mathrm{a})$. In addition, two splice variants with deletion of exon 5 (OPN-b) or exon $4(\mathrm{OPN}-\mathrm{c})$ have been described. Specific combinations of OPN splice variants are associated with distinct malignancies and different stages of cancer development and progression [11,28]. All three forms contain several highly conserved elements including an aspartic rich sequence at the N-terminal and, at the C-terminal, a calcium binding site and a heparin binding domain which is involved in the interaction with certain CD44 variants ( $v 6$ and/or v7). The central region of OPN contains a core block of integrin-binding sites that includes an RGD motif and a SLAYGLR domain (in rat and mouse OPN, SVVYGLR in human), which is followed by a thrombin cleavage site. RGD interacts with $\alpha v \beta 1-, \alpha v \beta 3-$ and $\alpha v \beta 5$-integrins while SLAYGLR, exposed by thrombin cleavage, binds to $\alpha 9 \beta 1-, \alpha 4 \beta 1-, \alpha 4 \beta 7$-integrins [7,29]. In addition to thrombin, other enzymes, such as matrix metalloproteinases (MMPs), can cleave OPN at specific sites thus modulating OPN functions and integrin binding properties. Increasing evidence suggests that the N- and C- terminal fragments generated by OPN proteolytic-cleavage, present in plasma and milk, may have physiologically distinct roles and acquire new functions compared to full-length isoform [6,30]. The thrombin-cleaved $\mathrm{N}$-terminal fragment is the predominant form in human bone marrow and, through its binding to $\alpha 9 \beta 1$ and $\alpha 4 \beta 1$ integrins, regulates hematopoietic progenitor cell homing and promotes IFN- $\gamma$ secretion in T cells $[10,31]$. The C-terminal fragment (OPN-CTF) is chemotactic for macrophages and inhibits IL-10 secretion by selectively interacting with CD44 isoforms [7,32]. On the other hand, the MMP9-cleaved isoform was recently shown to mediate tumor immune escape by inducing expansion of myeloid-derived suppressor cells (MDSCs) [33]. In addition, the MMP3/7-cleaveage of mouse OPN generates, in the $C$ terminal site, a novel $\alpha 9 \beta 1$ integrin binding motif $\left({ }^{152}\right.$ LRSKSRSFQVSDEQY $\left.{ }^{166}\right)$ involved in the development of antibodyinduced arthritis [34]. Besides proteolytic processing, post-translational modifications (such as phosphorylation, sulfation and glycosylation)

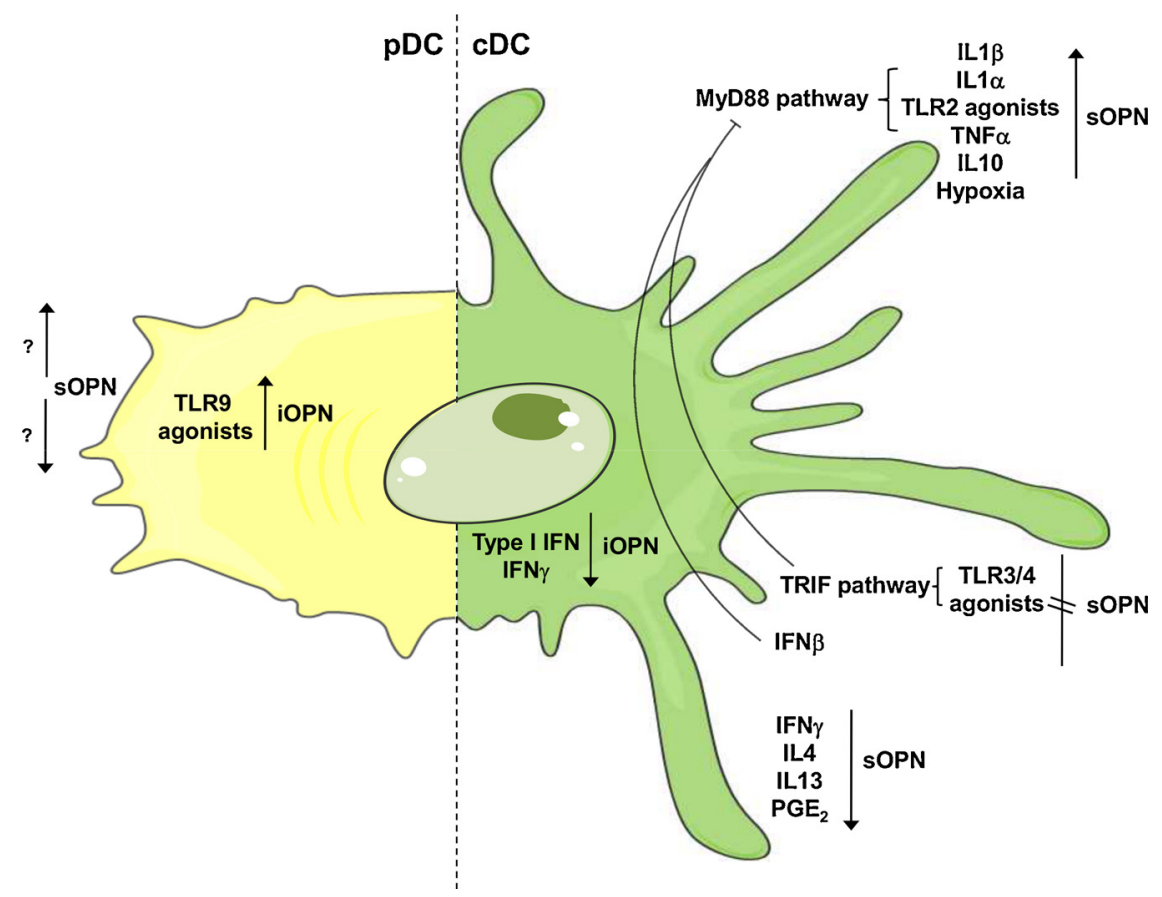

Fig. 1. Regulation of OPN production in DC subsets. Positive and negative mechanisms of regulation of sOPN and iOPN expression in DC subsets are summarized. iOpn is upregulated upon TLR9 engagement in pDCs (in yellow, left part of the Figure). Different pro- and anti-inflammatory stimuli are involved in the regulation of both sOPN and iOPN in CDCs (in green, right part of the Figure). 
Table 1

The autocrine/paracrine effects of OPN on DC functions.

\begin{tabular}{|c|c|c|}
\hline OPN effects on DC & Conditions and OPN type & References \\
\hline$\uparrow$ DC differentiation, survival and maturation induced by LPS & moDC; anti OPN Ab & [23] \\
\hline$\uparrow$ migration and MHC II molecules & LC in a skin explants model; rOPN & [25] \\
\hline $\begin{array}{l}\uparrow \text { MHC class II, costimulatory, and adhesion molecules; TNF } \alpha \text { and IL12 secretion; enhanced T-cell } \\
\text { allostimulatory capacity and IL12p70 secretion }\end{array}$ & moDC; rOPN & [25] \\
\hline Regulation of IL-12p70 production & LPS treated-BM-DC from OPN-/- and WT mice; rOPN & [69] \\
\hline$\downarrow D C$ maturation and cytokine production & $\begin{array}{l}\text { Human BDCA-1 + cells after HBV antigenic } \\
\text { stimulations; anti OPN Ab }\end{array}$ & [71] \\
\hline$\downarrow D C$ maturation and function, IL12 secretion & $\begin{array}{l}\text { BM-DC from OPN-/- and WT mice after HBV antigenic } \\
\text { stimulations }\end{array}$ & [71] \\
\hline$\uparrow$ maturation of DCs & $\begin{array}{l}\text { BDCA-1 + cells from CHB patients after HBcAg } \\
\text { stimulation; rOPN }\end{array}$ & [71] \\
\hline$\uparrow$ IFN $\alpha$ upon TLR9 stimulation & pDC in OPN-/- mice; iOPN & [49] \\
\hline$\downarrow$ IL27 production & $\mathrm{cDC}$ in $\mathrm{OPN}-/-$ mice; iOPN & {$[50]$} \\
\hline Emigration from epidermis & LC in OPN-/- mice; CHS model & [35] \\
\hline \multicolumn{3}{|l|}{$\uparrow$ MHC II molecules } \\
\hline$\uparrow$ OPN production, autocrine effect on migratory ability & $\begin{array}{l}\text { TNF } \alpha / \mathrm{IL} 1 \alpha \text { stimulated BM-DC from OPN-/- and WT } \\
\text { mice }\end{array}$ & [24] \\
\hline Different recruitment of DC subsets to regulate Th2 response & pDC and $\mathrm{cDC}$ in AHR mouse model; anti OPN Ab & [65] \\
\hline$\downarrow$ CCR5/CCR7 & In vivo $\mathrm{cDC}$ during immunization with listerial antigen & [59] \\
\hline$\downarrow$ migration to inflammatory site & and $\mathrm{rOPN}$ & \\
\hline \multirow[t]{2}{*}{$\uparrow \mathrm{CC}$ chemokine-induced migration } & $\begin{array}{l}\text { moDC and BM-DC from OPN-/- and WT mice; OPN } \\
\text { fragments }\end{array}$ & [36] \\
\hline & Thrombin cleaved fragment & \\
\hline$\uparrow$ CCR7 & pDC in mouse model of asthma; OPN SLAYGLR motif & [37] \\
\hline$\uparrow \operatorname{IFN} \beta$ & & \\
\hline$\uparrow$ recruitment durin & & \\
\hline
\end{tabular}

Abbreviations: rOPN, recombinant osteopontin; iOPN, intracellular OPN; BM-DC, Bone-Marrow DC; moDC, monocyte derived DC; HBcAg, Hepatitis B core Antigen; pDC, plasmacytoid DC; cDC, conventional DC; CHB, chronic Hepatitis B; CHS model, Contact Hypersensitivity Syndrome model; AHR, airway hyperresponsiveness.

regulate many biological OPN functions [26].

The outcome of OPN signaling depends on many different conditions, such as cell type, receptor engaged, homeostatic/inflammatory environment that determines OPN cleavage and levels of receptor expression. Integrins and CD44 isoforms, recognized as OPN receptors, are expressed by a wide array of both non-immune and immune cells, including DCs. Different receptor combinations were described to mediate OPN-induced DC functions, based on the activation context. OPN was described to induce LC migration by interacting with CD44 and $\alpha v \beta$ integrins [35]. On the contrary, no involvement of CD44 was apparently required in OPN mediated $\mathrm{CDC}$ and $\mathrm{pDC}$ migration [36,37].

To add more complexity to OPN mechanism of action, mouse OPN mRNA contains an alternative translational start site that allows the generation of a shorter form of OPN. This form lacks the N-terminal signal sequence and is retained intracellularly (iOPN), in the cytoplasm or nucleus [8]. iOPN, originally identified in rat calvarial cells, plays a role in the proliferation and motility of various cell types (e.g. fibroblasts, osteoclasts, macrophages) by interacting with the CD44-ERM(ezrin-radixin-moesin-) actin complexes [26,38]. Growing evidence supports also a role of iOPN in immune responses. Indeed, iOPN regulates follicular T cell differentiation [39] and NK cell activation [40]. In murine macrophages, iOPN contributes to antifungal and antiviral immunity by interacting with TLR2 and dectin-1 [41] and stabilizing TRAF3 [42]. Alternatively, in pDCs, iOPN cooperates with MyD88 in TLR9 signaling. Recent evidence also indicates that the coordinated expression of sOPN and iOPN can shape emergency mouse hematopoiesis and regulate the functions of DC subsets [43]. Although iOPN was detected in human cell lines by confocal microscopy $[8,44]$, the current understanding of iOPN expression and function in human cells is still limited.

\section{Regulation of OPN production in DCs}

OPN mRNA and protein secretion are upregulated early during differentiation of DC progenitors, such as $\mathrm{CD} 14^{+}$monocytes and BM precursors [23], and immature DCs produce conspicuous amounts of OPN [24]. Different stimuli were shown to potentiate the release of
OPN during DC differentiation. For example, 9cis-Retinoic acid strongly enhances OPN secretion during the differentiation of murine DCs [45]. Likewise, hypoxia significantly increases the production of OPN in both immature and mature DCs with obvious implications for the pathogenesis of tumors, inflammatory and autoimmune diseases [46,47].

Maturation is a complex process that can confer distinct functional properties to DCs. Pathogen associated molecular patterns (PAMPs) and inflammatory stimuli (e.g. TNF- $\alpha$, IL-1 $\beta$, and type I and type II IFNs) activate DCs to induce specific immune responses. However, under the influence of anti-inflammatory soluble factors such as TGF $\beta$, IL-10, IL-4, PGE2, DCs acquire regulatory functions leading to Treg differentiation. These different two classes of signals also regulate OPN expression.

\subsection{PAMPS and inflammatory cytokines}

To recognize pathogen signatures DCs express an array of pattern recognition receptors, which includes toll-like receptors (TLRs). The engagement of specific TLRs differentially impacts on OPN production. TLR2 agonists, such as Staphylococcus aureus, are strong OPN inducers. Conversely, TLR3 and TLR4 agonists, such as Poly I:C and LPS, do not induce OPN production [24,48]. In general, OPN is preferentially induced by TLRs that signal exclusively through MyD88 pathway [48]. TLR3 and TLR4 agonists, although inactive by themselves, negatively regulate TLR2-dependent OPN production possibly inducing a TRIFdependent pathway which interferes with MyD88-dependent OPN upregulation [48]. Pro-inflammatory cytokines, such as IL-1 $\beta, \mathrm{IL}-1 \alpha$ and TNF- $\alpha$ were also reported to upregulate OPN secretion [24].

Relevant aspects concerning the regulation of iOPN and sOPN by TLR ligands have been described in mouse DC subsets [49]. Although both mouse pDCs and cDCs express TLR9, CpG selectively stimulates iOPN in pDCs, by a T-bet dependent mechanism. In these cells, iOPN colocalizes with the TLR9-MyD88-associated signaling complex, leading to IRF7 nuclear translocation and IFN- $\alpha$ gene transcription [49]. In a complex interplay between pDCs and cDCs, it was shown that pDCderived IFN- $\alpha$ inhibits iOPN production by cDCs [50]. Similarly, IFN- $\gamma$, long being considered as a proinflammatory cytokine, limits OPN expression in cDCs both in vitro and in vivo [51]. This inhibitory effect 
supports the emerging view that IFN- $\gamma$, at the peak of inflammation, may exert regulatory functions to control tissue damage. OPN regulation by type I and type II IFNs may have important implications in the development of Th17 cells in autoimmune inflammation.

\subsection{Anti-inflammatory mediators}

The Th2 cytokines IL-4 and IL-13, known for their negative regulation of DC functions, were reported to inhibit OPN secretion in monocyte-derived DCs [52]. OPN was otherwise induced by IL-10 both in DCs [52] and in macrophages where it was associated with cardiac fibrosis [53].

Eicosanoids, including $\mathrm{PGE}_{2}$, may also differently affect DC functions depending on the nature of maturation signals and tissue localization. PGE $_{2}$ exerts immunosuppressive activity by upregulating IL-10 production and dampening the secretion of pro-inflammatory cytokines and chemokines (e.g. IL-12, TNF- $\alpha$, CCL3, and CCL4) [54,55]. Similarly, $\mathrm{PGE}_{2}$ and MSC-derived $\mathrm{PGE}_{2}$ suppress OPN production by DCs [56].

\section{Autocrine/paracrine effects of DC-derived OPN: role in shaping immunity}

\subsection{Effects of OPN on DC migration}

DC migration is a crucial process implicated in induction and regulation of immune responses both in steady-state and pathological conditions. OPN was described to regulate DC migration at multiple levels [57,58]. Initial evidence reported that OPN regulates the migration of LCs to draining cutaneous lymph nodes during the sensitization phase of contact hypersensitivity [35]. As a result, OPN deficient mice were defective in LC migration and in mounting delayed-type hypersensitivity responses. The chemotactic effect of OPN was mediated by the OPN receptors CD44 and $\alpha v \beta 3$ expressed by DCs [35].

OPN may regulate DC migration also by an autocrine mechanism. Indeed, the induction of OPN by different stimuli, such as TNF- $\alpha$, CCL19 or OPN itself was able to improve DC motility.On the other hand, reduction of DC migration was observed upon LPS stimulation, a condition known to down-regulate OPN expression [24]. The mechanism by which endogenous OPN expression promotes DC migration is still unclear and it might involve iOPN contribution. Most of the studies on OPN chemotactic activity were performed with the fulllength OPN, that contains several cellular binding domains responsible for cell adhesion, spreading, and migration. The OPN fragments, generated by thrombin and carboxypeptidase cleavage, although not directly chemotactic, were shown to potentiate DC migration driven by CCL21 and CCL19 through RGD domain and the exposure of a new prochemotactic sequence [36]. These results candidate OPN as a modulator of DC migration in inflammatory conditions.

Although the pro-migratory activity of OPN is largely demonstrated, evidence of an inhibitory effect of OPN on DC migration was also reported in a model of immunization with listerial antigens. Exogenous OPN was found to suppress migration of cDCs in vivo, from the site of bacteria inoculation to draining lymph nodes, through the down-regulation of the chemokine receptors CCR5 and CCR7 [59]. CCR5 is the main chemokine receptor that regulates the migration of immature DC into tissues under inflammatory conditions, whereas CCR7 directs activated DCs to lymph nodes to activate specific immune responses [60]. The modulation of chemokine receptors is a recognized mechanism of regulation for DC trafficking [57] and OPN is emerging as a new molecule involved in this complex signal network. By differentially promoting the migration of DC subsets OPN may regulate Th2 responses [61]. During allergic airways responses, pDCs play a tolerogenic role through the production of type I interferon and the induction of Treg cells. Conversely, cDCs promote Th2 responses and airway inflammation [62-64]. In a model of ovalbumin-induced airway hyperesponsiveness, sOPN was shown to exert opposite effects by the differential recruitment of pDCs and cDCs to the site of antigen response [65]. In primary systemic immunization, sOPN neutralization was associated with enhanced recruitment of tolerogenic pDCs into the draining lymph nodes and reduction of Th2 allergic response [65]. On the contrary, the neutralization of sOPN during secondary antigen challenge, promoted the recruitment of $\mathrm{cDCs}$ leading to the alteration of the $\mathrm{cDC} / \mathrm{pDC}$ ratio and the exacerbation of the phenotype [65]. The intranasal administration of recombinant OPN during antigen challenge reversed the phenotype and protected from allergic inflammation, suggesting a therapeutic potential of recombinant OPN in allergic airway inflammation [66].

An additional evidence for the role of OPN in DC migration was recently reported in tolerance induction [37]. The injection of OPN along with endotoxin-free antigen, prior to the sensitization phase of an asthma model, generated an immunoregulatory microenvironment in the draining lymph node, through the accumulation of Foxp $3^{+}$Treg cells. Foxp $3^{+}$depletion abolished OPN-induced tolerance. Moreover, pDC depletion hampered OPN-mediated increase of Foxp $3^{+}$Treg cells in mediastinal lymph nodes, suggesting that the tolerogenic action of OPN is mediated by pDC accumulation [37]. OPN-induced recruitment of pDCs during the tolerance phase is mediated by the upregulation of CCR7 and the increased transcription of the CCR7 ligands CCL19 and CCL21 in regional lymph nodes. The thrombin-cleaved SLAYGLR motif was identified as responsible for the recruitment of tolerogenic pDCs. However, the integrin expressed by pDC, responsible for the interaction of OPN with the SLAYGLR motif, still needs to be characterized [7].

These results suggest that sOPN can regulate the immune response in asthma by selectively affecting the recruitment of cDC and/or pDC subsets at different phases of the allergic reaction and candidate OPN for future development of targeted immunotherapies (Fig. 2).

\subsection{Effects of DC-derived $O P N$ on Th1 and Th2}

OPN was initially identified as a $\mathrm{T}$ cell factor playing a central role in the induction of Th1-mediated responses and the inhibition of Th2associated cytokine expression. OPN deficient mice have severely impaired type- 1 immunity to viral and bacterial infections and develop sarcoid-type granulomas [4]. Dysregulated OPN overexpression was instead correlated with Th1-related disorders, such as EAE/Multiple Sclerosis, rheumatoid arthritis and Crohn's disease [6,9,67]. OPN promotes Th1 polarization mainly through an effect on DCs. Indeed, by acting in an autocrine/paracrine manner, OPN contributes to DC maturation and survival [23]. OPN also triggers TNF- $\alpha$ and IL-12 production and DC shaping toward a Th1-polarized phenotype. These observations make OPN a central player in DC recruitment and activation during the sensitization phase in Th1-driven contact hypersensitivity reactions [25]. OPN, highly secreted by effector T cells and keratinocytes, may also contribute to chronification of contact hypersensitivity reactions perpetuating Th1 response, attracting inflammatory cells and sustaining IL-12 production by DCs and macrophages [68]. DC-derived OPN might also regulate the Th1/Th2 balance and limit the extent of the Th2 and IgE response as shown in a mouse model of systemic allergen sensitization [69]. A role of OPN in promoting a protective Th1 response and in the generation of Treg cells was also proposed in patients undergoing allergen immunotherapies (e.g. venom immunotherapy) [70].

DC-derived OPN plays also a protective role in viral infections. Exposure of DCs to HBV antigens induces high levels of OPN and promotes DC maturation, IL-12 secretion and Th1 response. In agreement with this result is the observation that DCs from patients with chronic hepatitis $\mathrm{B}$, that secrete lower levels of OPN compared to normal controls, have a defect in Th1 response to HBV antigens. This finding is further supported by the evidence that DCs obtained from OPN deficient mice exposed to HBV antigens mount reduced inflammatory and IFN- $\gamma$ responses [71]. However, during viral infections, also pDCs can contribute to Th1 responses through the production of IFN- $\alpha$ and IFN- $\gamma$ 


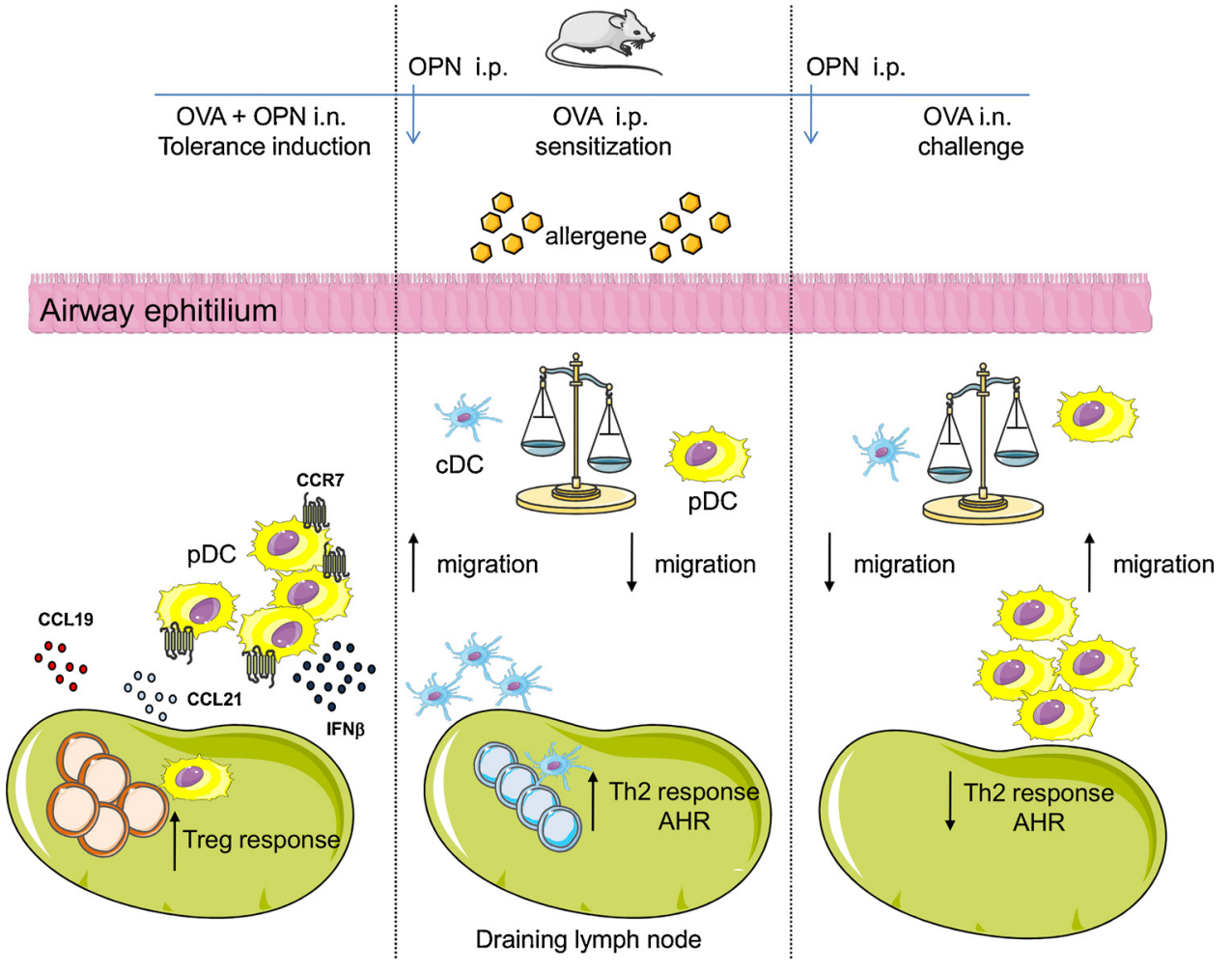

Fig. 2. Role of OPN on $\mathrm{pDC} / \mathrm{cDC}$ balance and $\mathrm{T}$ cell polarization in allergic airway disease. During antigenic tolerance induction (in the left), prior to sensitization phase, the injection of OPN, along with endotoxin free antigen, induces immune suppressive microenvironment in the draining lymph node through the accumulation of Treg, which is mediated by pDC recruitment. OPN induces upregulation of CCR7 on pDCs and increases transcription levels of the CCR7 ligands CCL19 e CCL21 in regional lymph node [37]. During the sensitization phase (in the center), OPN plays a proinflammatory role through the inhibition of tolerogenic pDCs in the regional lymph node and enhances inflammatory AHR responses. During secondary antigenic challenge (in the right), OPN inhibits the migration of cDCs into draining lymph node, alters the cDCs:pDCs balance and reduces Th2 responses and AHR reactions [65]. induction in T cells [72,73]. In this context, iOPN might be critical for TLR9-mediate IFN- $\alpha$ stimulation, whereas sOPN is apparently dispensable [49].

A number of studies have also recognized an important role of OPN in Th2-associated diseases. Increased OPN levels were detected in the airways, serum and biological fluids of patients with asthma [74], allergic ocular diseases [75] and rhinosinusitis [76]. Nevertheless, the contribution of OPN in Th2 immunity remains to be fully elucidated as OPN effect varies depending on the type and phase of allergic contact dermatitis and allergic disease. For instance, in animal models, the use of an OPN neutralizing antibody before the sensitization phase (or the secondary challenge), promotes a Th2 response during allergen-sensitization, while it inhibits Th2 response during allergen challenge [65]. In addition, discrepancies were described between results obtained with the use of blocking antibodies and OPN deficient mice suggesting that timing in measuring IgE levels, strain of mice and specific functions of iOPN vs. sOPN might be relevant for the response $[66,69,74]$. Finally, when considering the role of OPN in Th2 response, it is critical to consider that DCs, although fundamental, need to be supported by other cell types, such as epithelial cells, basophils and mast cells for the induction of Th2 responses [77,78]. Some of these cells also produce OPN and can contribute in shaping the immune response to allergens. For example, epithelial cells triggered by allergens were shown to produce OPN and Th2-promoting cytokines such as IL-33, thymic stromal lymphopoietin (TSLP) and IL-25. Of note, OPN is produced by mast cells and promotes mast cell migration and IgE-mediated degranulation [79].

\subsection{Effects of DC-derived OPN on Th1/Th17}

Th17 cells mediate antimicrobial inflammatory responses against extracellular bacteria, particularly those that colonize airways, skin, and intestinal lumen through activities on immune and nonimmune cells [80]. in vitro experiments have shown that OPN produced by $S$. aureus-treated DCs promotes IL-17 production by $\mathrm{CD}^{+}{ }^{+} \mathrm{T}$ cells [81]. Accordingly, a connection between TLR2 and a robust Th17 activation by gram-positive bacteria was found in the response to $M$. tuberculosis infection [82] and Th17 cells seem to be essential in the clearance of both $S$. pneumoniae and $S$. aureus [83,84]. However, specific expression of the two OPN isoforms by DC-subsets appears to be fundamental in Th1/Th17 development in the context of inflammatory/autoimmune diseases [8].

\subsection{1. $E A E / M S$}

A role for DC-derived OPN in IL-17 production was demonstrated in both EAE and MS. Furthermore, OPN receptors were upregulated in T cells during the development of the disease [85]. Studies in experimental models, provided evidence on the role of iOPN expression by cDCs (and microglia) in Th17 commitment in vitro and in vivo and in the regulation of EAE onset and severity [50]. Expression of iOPN by cDCs inhibits IL-27 production and promotes the response of Th17 cells. Consistent with these results, a delay in EAE onset was observed in OPN-deficient mice and this phenotype is associated with an increase in IL-27 expression. In addition, IFNAR signaling can suppress Th17 differentiation by inhibiting iOPN, thus providing insights into the therapeutic impact of type I IFN treatment of MS and other Th17-driven diseases. Since OPN expression is induced by TLR engagement in pDCs but not cDCs [49], it is possible that the IFN- $\alpha$ produced by pDCs may negatively regulate Th17 generation through engagement of IFNAR expressed by cDCs.

\subsubsection{Psoriasis}

Both cDC- and pDC-derived OPN can contribute to the pathogenesis of Th1/Th17-mediated psoriatic disease [86]. In human psoriatic skin, an overall increase of DCs is found both in the epidermis and in the dermis [87]. Dermal DCs may be involved in the early phase of psoriasis via production of IL-23 that mediates expansion of Th17 and Th22 cells [88]. In addition, pDCs are an important initiator of the psoriatic process through the production of IFN- $\alpha[87,89]$. Immunohistochemical staining of psoriatic plaque biopsies revealed that OPN is highly expressed by keratinocytes, endothelial cells and immune infiltrating cells, including $\mathrm{CD} 1 \mathrm{a}^{+}$DC and effector T lymphocytes [86]. Moreover, 
increased OPN plasma levels was found associated with psoriasis [90]. Based on this evidence, it was proposed that, in genetic susceptibility conditions, the expression of OPN, triggered by bacteria or trauma, may enhance DC/LC migration and Th1 polarization. pDCs can be recruited to the inflammatory site upon TLR activation and contribute to Th1 responses through iOPN overexpression and IFN- $\alpha$ production. On the other hands, iOPN expressed by activated cDCs could drive Th17 polarization by the inhibition of IL-27 expression [50]. In this context, OPN might directly influence the production of IL- 17 by CD4 ${ }^{+} \mathrm{T}$ cells. The chronic psoriatic inflammation is additionally sustained by the prosurvival activity of OPN on keratinocytes and by the OPN-mediated proangiogenic effects, which promote new vessel formation and recruitment of immune cells. Therefore, OPN is part of the complex cytokine network that stimulates inflammatory response in psoriasis and may represent a new therapeutic target in this disease.

\subsection{3. $C O P D / I B D$}

Chronic obstructive pulmonary disease (COPD) and inflammatory bowel diseases (IBD) are chronic inflammatory diseases of mucosal tissues that affect the respiratory and gastrointestinal tracts, respectively. COPD and IBD share many epidemiological and clinical similarities [91] and represent systemic inflammatory diseases in which OPN and Th1/Th17 may contribute to pathogenesis. Recently, it was reported that, during intestinal inflammation, elevated levels of OPN are secreted by $\mathrm{CD}_{103^{-}}$DCs and contribute to their capacity to secrete proinflammatory cytokines and induce Th1/Th17 responses. This effect was shown to be mediated by the interaction of the thrombin exposedSLAYGLR OPN domain with $\alpha 9$ integrin expressed on CD103- DC [92].

The presence of Th1/Th17 biased T cells is important in the development and progression of airway inflammation and COPD. OPN deficient mice were protected against cigarette smoke-induced disease and their lung DCs failed to promote Th1 or Th17 cell differentiation in vitro [93]. The transcriptome analysis of lung DCs from OPN deficient mice exposed to cigarette smoke showed that several IFN-stimulated genes, including Irf7, were up-regulated in the absence of OPN, suggesting that OPN plays its effect at least in part through the inhibition of Irf7 expression, a transcription factor critical for limiting pro-inflammatory cytokine production. Accordingly, reduction of Irf7 expression restored the functions of OPN deficient lung DCs required for Th17 cell differentiation [93].

\subsection{Effects of $D C$-derived $O P N$ on Treg cells}

DC-derived OPN plays an important role in Treg differentiation and accumulation. Evidence shows that the enhanced secretion of OPN by DCs differentiated in the presence of 9-cis retinoic acid (9cisRA-DC) is important for the conversion of naïve T cells into Tregs [45]. 9cisRA-DC $\left(\mathrm{CD} 103^{-} \mathrm{CD}^{207^{-}}\right.$) expressed low levels of MHC II and costimulatory molecules but high levels of the inhibitory molecule PD1-L and efficiently induced Tregs. 9cisRA-DCs from OPN deficient mice were compromised in the ability to induce Treg cells and this defect could be restored by recombinant $\mathrm{OPN}$, in vitro. In vivo injection of antigenpulsed 9cisRA-differentiated DCs inhibited CHS response, thus confirming their tolerogenic potential. This result suggests that the induction of Treg cells by DC-derived OPN might contribute to the therapeutic effects of 9cisRA, a recent option for the treatment of immune mediated skin diseases [94].

Moreover, retinoic acid is crucial for several of the functions mediated by the intestinal immune system where DCs play a crucial role in the maintenance of tolerance to commensal bacteria and food antigens [95]. Further studies will clarify the influence of OPN in the Treg/ $\mathrm{T}$ effector cell balance and in oral tolerance [96]. A role for OPN as a tolerance enhancer was recently reported in allergic airway disease, where, OPN is responsible for the accumulation of Foxp $3^{+}$Treg lymphocytes, through the recruitment of anti-inflammatory CCR7 expressing pDCs [37].

\section{DC-derived OPN in tumor progression}

OPN represents one of the best characterized matricellular proteins related to tumor metastasis and proliferation [10,11]. High levels of OPN, in blood and in tumors of cancer patients, correlated with a poor prognosis. These observations suggest that OPN may represent an important diagnostic and prognostic marker in different tumor histotypes [29]. From a pathogenic/mechanistic point of view, OPN is involved in several steps of tumor biology and progression, such as proliferation, survival, angiogenesis, and invasion (such as in breast, lung, prostate, colorectal, hepatic cancers, melanoma and mesothelioma) [10,97-99].

In the tumor microenvironment OPN can be produced by neoplastic cells as well as by infiltrating immune cells, including DCs, tumor-associated macrophages (TAMs) and Myeloid-Derived Suppressor Cells (MDSCs) [100-102]. Several studies show that OPN creates a protumorigenic microenvironment by different mechanisms. For example, TAMs interacting with colorectal cancer stem cells by secreting OPN, promote tumorigenicity and clonogenicity [103]. OPN has also a role of in the expansion and activitiy of MDSCs [104,105]. In a mouse model of metastatic breast cancer, both tumor- and myeloid-derived OPN contribute to metastatic progression. Tumor cells express s OPN that promotes survival and support the production of VEGF and IL-6, which, in turn, can increase their suppressive activity of MDSCs sustaining the expression of Arg1 and Nos2 [106]. On the other hand, M-MDSCs express mainly iOPN that is involved in the maintenance of their suppressive activity. OPN expressed by MDSCs and tumor cells was demonstrated to suppress the activity of cytotoxic $\mathrm{CD} 8^{+} \mathrm{T}$ lymphocytes by binding to CD44, thereby contributing to tumor progression. OPN expression is suppressed by the binding of interferon regulatory factor 8 (IRF8) to the Spp1 promoter. Accordingly, IRF8 silencing in MDSCs during cell transformation is associated with OPN upregulation [107]. This study provides a perspective on OPN/CD44 interaction as an additional immune checkpoint that can be exploited to enhance immune responses against tumors.

DCs have been long considered as a critical component of antitumor immunity, in particular, by their capacity to cross-present self-tumor antigens to $\mathrm{CD}^{+} \mathrm{T}$ cells. However, in the tumor context, DCs can be severely limited in their differentiation and activation and are poor stimulators of immune responses [108,109]. Among the mechanisms of negative regulation of DC functions, hypoxia, which characterizes the tumor microenvironment, impairs DC maturation and induces their transcriptional reprogramming $[46,110]$. OPN was identified as one of the most hypoxia-regulated gene [46] and hypoxic DCs produce increased OPN levels, which play an important role in enhancing tumor cell migration [47].

These results suggest that in the tumor microenvironment OPN has different functions in relation to producer cell types and emphasize the role of DC-derived OPN in the metastatic phenotype of cancer cells.

\section{Role of OPN in MSC/DC functional interaction}

Mesenchymal Stem Cells (MSCs) were first characterized in bone marrow (BM), where they are involved in the maintenance of the BM niche by inhibiting the differentiation of hematopoietic stem cells (HSCs) by cell-to-cell contact and the release of soluble factors, including angiopoietin-1, thrombospondin-1 and 2 and OPN. Moreover, for their ability to differentiate into diverse cell lineages, MSCs have long been proposed to be the in vivo precursors of some non-hematopoietic components of the BM, such as osteoblasts, adipocytes and fibroblastic reticular cells. BM-resident MSCs are positioned in perivascular areas of BM microenvironment, where they can be in close contact with immune cells, including B cells, T cells and DCs [111].

A number of in vitro studies have described the effect of MSC/DC interaction [20,112-114]. Recent evidence demonstrates that DCs influence MSC behavior and that DC-derived OPN contributes to the biological relevance of DC/MSC cross-talk [56]. Resting MSCs promote 
OPN production by DCs and DC-conditioned medium enhances osteogenic differentiation of MSCs. This process is associated with the upregulation of the osteogenic markers alkaline phosphatase, RUNX2, and the expression of the bone-anabolic chemokine CCL5. In contrast, OPN may play an inhibitory role in adipogenic differentiation as indicated by the downregulation of PPAR $\gamma$, adiponectin, and FABP4. These effects can be reduced by the presence of RGD peptides or antibodies against integrin $\beta 1$, unveiling a role for DC-derived OPN in the regulation of MSC differentiation [56]. The capacity of OPN to interfere with adipocyte/osteoblast development and functions is also supported by the findings that OPN deficiency causes delayed bone formation by MSCs and promotes adipogenesis in vivo [115]. In addition, DC-derived extracellular vesicles containing OPN were reported to mediate MSC recruitment [116], suggesting a more complex role for DC-derived OPN as a chemoattractant and modulator of MSC differentiation. Accordingly, OPN was shown to regulate MSC migration and differentiation in multiple cell types during skin wound healing [117].

The discovery of the immunomodulatory properties of MSCs [118] has generated growing interest for these cells as a new potential therapeutic strategy in chronic inflammation and autoimmune disorders [119]. MSCs home to active inflammatory sites [120] where they suppress the differentiation/maturation of DCs, the proliferation of $\mathrm{T}$ cells and the release of cytokines by both cell contact-dependent and -independent mechanisms [121]. The activation of MSCs with proinflammatory cytokines (i.e., TNF- $\alpha$, IL-6, and IL-1 $\beta$ ) in vitro, was shown to suppress the production of DC-derived OPN with $\mathrm{PGE}_{2}$ being the main soluble factor responsible for this inhibition [56]. The ability of $\mathrm{PGE}_{2}$ to reduce OPN levels was implicated in vivo in the protection provided by $\mathrm{PGE}_{2}$ in allergic asthma. In mice, intranasal $\mathrm{PGE}_{2}$ administration prevented hyperresponsiveness to aeroallergen through reduction of OPN that likely allows the recruitment of tolerogenic pDCs [122]. Based on these observations, the regulation of OPN levels might represent one of the mechanisms underlying the beneficial effects of MSC in airway inflammation and other inflammatory diseases [123]. These results suggest that, in physiological conditions, or at the beginning of an inflammatory response, MSCs could potentially stimulate resident DCs to produce OPN, responsible for the recruitment of additional immune cells. OPN can also promote the recruitment of MSCs through the upregulation of CCL5. On the contrary, at later stages of inflammation, activated MSCs, through the production of $\mathrm{PGE}_{2}$, downregulate OPN secretion and limit inflammatory events (Fig. 3).

\section{Concluding remarks}

OPN is a pleiotropic cytokine, highly conserved in evolution [1], that acts through the activation of multiple receptors, expressed by different cell types. For these reasons, OPN is associated with a complex variety of biological functions and is involved in several biological aspects, ranging from regulation of normal immune responses, inflammation, infections, autoimmune diseases and cancer, and its function is influenced by tissue and context of action. OPN has also been recently proposed as a therapeutic target to control tumor growth, asthma and autoimmune diseases, as well as a promising modulator of immune ontogeny in infancy. OPN neutralization for the treatment of autoimmune disorders has been pursued in several preclinical studies as well as in clinical trials. In addition, OPN single nucleotide polymorphisms (SNPs) and OPN variants are explored as a diagnostic target to monitor several immune mediated conditions. Interestingly, recent data show that the production of anti-OPN autoAbs may favour remission in both MS and EAE, which leads to novel strategies, such as vaccination or passive immunization, to boost their levels [11,28,124-127].

DCs represent an important source as well as a target of OPN action being regulated in several aspects of DC biology, including migration, maturation and cytokine secretion. An important aspect of DC-OPN interplay is related to the heterogeneity of DC subsets and functions. Similarly, the existence of different OPN isoforms and cleavage products introduces further levels of regulations and plasticity in the crosstalk between DCs and OPN. A still unresolved aspect is related to the understanding of OPN regulation of human responses in relation to the different roles played by sOPN vs. iOPN, an aspect so far documented only in the mouse system. A major interest for the role of OPN in shaping immune response is the ability to regulate cytokine production by DCs, thus influencing $\mathrm{T}$ cell polarized responses. In addition, OPN released by DC can mediate tumor progression acting directly on tumor cell proliferation, survival and dissemination or indirectly contributing to the generation of a permissive microenvironment through the recruitment of myeloid-derived suppressive cells.

Finally, an emerging role of OPN in inflammation has been recently proposed on the basis of the bidirectional role of this cytokine in DC/ MSC interaction, both in the bone marrow and the inflammatory sites. MSCs are in the limelight of cell-therapy for their potential immunomodulatory function in immune-mediated degenerative pathologies, such as rheumatoid arthritis, osteoarthritis, multiple sclerosis, and for the control of graft-versus-host diseases following bone marrow transplantation [112,128]. Evidence suggests that MSC participate to

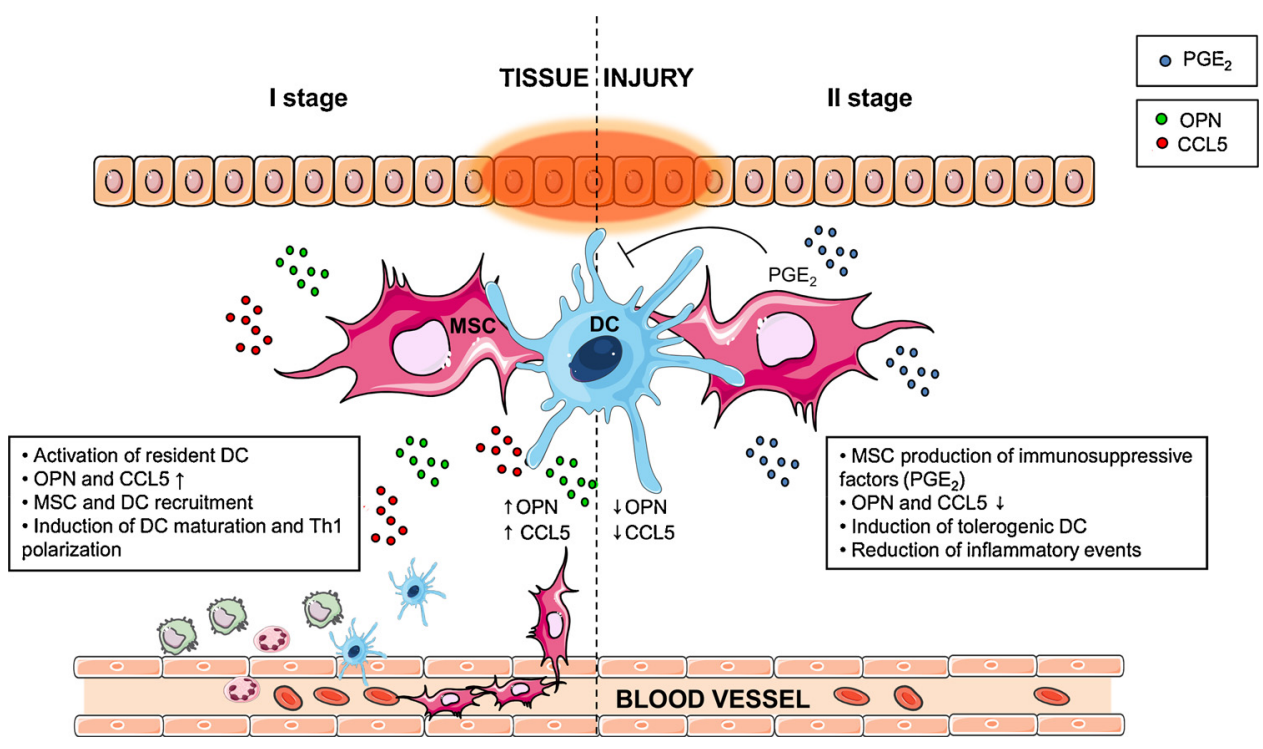

Fig. 3. Role of OPN in DC/MSC interaction. A schematic representation of the interaction between DCs and MSCs during inflammatory conditions. During early phase of inflammation (I stage, left part of the Figure), the interplay between DCs and MSCs at the injury site may contribute to the upregulation of OPN production that supports further attraction of MSCs and DCs from peripheral circulation. Simultaneous increase of CCL5 promotes the recruitment of inflammatory cells. While the inflammatory response evolves (II stage, right part of the Figure), MSCs, exposed to sufficient levels of proinflammatory cytokines, adopt an immune-suppressive phenotype and release $\mathrm{PGE}_{2}$ that inhibits OPN and CCL5 production by DCs. 
the regulation of inflammation in peripheral tissues [129]. In this regard, OPN may either directly promote MSC recruitment to injured sites where these cells may exert anti-inflammatory potential through the production of soluble mediators, such as PGE2, or can support further attraction of DCs from peripheral circulation [56]. A note of concern however comes from recent studies suggesting that MSCs may also contribute to tumor pathogenesis [130]. The relevance of DC/OPN crosstalk still needs to be better elucidated before being exploited and tested for new therapeutic strategies. Finally, the role of DC-derived OPN in the osteogenic versus adipogenic differentiation of MSCs might deserve to be investigated in the context of regenerative medicine [115]. All in all, OPN should be considered as a relevant DC effector cytokine involved in the plasticity that characterizes DCs behaviour in homeostatic and pathological immune responses.

\section{Declarations of interest}

All the authors declare no conflict of interest.

\section{Acknowledgments}

This work was supported by the Italian Association for Cancer Research (AIRC) under IG 2017 - ID. 20,776 project - P.I. Sozzani Silvano", by Fondazione Berlucchi, and by the Compagnia di San Paolo, Turin, Italy.

\section{References}

[1] G.F. Weber, The phylogeny of osteopontin-analysis of the protein sequence, Int. J. Mol. Sci. (2018) 19.

[2] A. Franzen, D. Heinegard, Isolation and characterization of two sialoproteins present only in bone calcified matrix, Biochem. J. 232 (1985) 715-724.

[3] D.R. Senger, D.F. Wirth, R.O. Hynes, Transformed mammalian cells secrete specific proteins and phosphoproteins, Cell 16 (1979) 885-893.

[4] S. Ashkar, G.F. Weber, V. Panoutsakopoulou, M.E. Sanchirico, M. Jansson, S. Zawaideh, et al., Eta-1 (osteopontin): an early component of type-1 (cellmediated) immunity, Science 287 (2000) 860-864.

[5] M. Singh, S. Ananthula, D.M. Milhorn, G. Krishnaswamy, K. Singh, Osteopontin: a novel inflammatory mediator of cardiovascular disease, Front Biosci 12 (2007) 214-221.

[6] S.R. Rittling, R. Singh, Osteopontin in immune-mediated diseases, J. Dent. Res. 94 (2015) 1638-1645.

[7] K.X. Wang, D.T. Denhardt, Osteopontin: role in immune regulation and stress responses, Cytokine Growth Factor Rev. 19 (2008) 333-345.

[8] M. Inoue, M.L. Shinohara, Intracellular osteopontin (iOPN) and immunity, Immunol. Res. 49 (2011) 160-172.

[9] T. Uede, Osteopontin, intrinsic tissue regulator of intractable inflammatory diseases, Pathol. Int. 61 (2011) 265-280.

[10] L.M. Castello, D. Raineri, L. Salmi, N. Clemente, R. Vaschetto, M. Quaglia, et al. Osteopontin at the crossroads of inflammation and tumor progression, Mediators Inflamm. 2017 (2017) 4049098.

[11] C. Hao, Y. Cui, S. Owen, W. Li, S. Cheng, W.G. Jiang, Human osteopontin: potential clinical applications in cancer (Review), Int. J. Mol. Med. 39 (2017) 1327-1337.

[12] S.G. Solano-Galvez, S.M. Tovar-Torres, M.S. Tron-Gomez, A.E. Weiser-Smeke, D.A. Alvarez-Hernandez, G.A. Franyuti-Kelly, et al., Human dendritic cells: ontogeny and their subsets in health and disease, Med. Sci. Basel (Basel) (2018) 6 .

[13] M. Collin, V. Bigley, Human dendritic cell subsets: an update, Immunology 154 (2018) 3-20.

[14] C. Macri, E.S. Pang, T. Patton, M. O'Keeffe, Dendritic cell subsets, Semin. Cell Dev. Biol. 84 (2018) 11-21.

[15] R.M. Steinman, H. Hemmi, Dendritic cells: translating innate to adaptive immunity, Curr. Top. Microbiol. Immunol. 311 (2006) 17-58.

[16] A.L. Blasius, M. Colonna, Sampling and signaling in plasmacytoid dendritic cells: the potential roles of Siglec-H, Trends Immunol. 27 (2006) 255-260.

[17] S. Sozzani, W. Vermi, A. Del Prete, F. Facchetti, Trafficking properties of plasmacytoid dendritic cells in health and disease, Trends Immunol. 31 (2010) 270-277.

[18] K. Clayton, A.F. Vallejo, J. Davies, S. Sirvent, M.E. Polak, Langerhans CellsProgrammed by the epidermis, Front. Immunol. 8 (2017) 1676.

[19] S.C. Eisenbarth, Dendritic cell subsets in T cell programming: location dictates function, Nat. Rev. Immunol. (2018).

[20] J.M. Ethokic, S.Z. Tomic, M.J. Colic, Cross-talk between mesenchymal Stem/ Stromal cells and dendritic cells, Curr. Stem Cell Res. Ther. 11 (2016) 51-65.

[21] M. Svensson, P.M. Kaye, Stromal-cell regulation of dendritic-cell differentiation and function, Trends Immunol. 27 (2006) 580-587.

[22] B. Pulendran, H. Tang, T.L. Denning, Division of labor, plasticity, and crosstalk between dendritic cell subsets, Curr. Opin. Immunol. 20 (2008) 61-67.

[23] K. Kawamura, K. Iyonaga, H. Ichiyasu, J. Nagano, M. Suga, Y. Sasaki, Differentiation, maturation, and survival of dendritic cells by osteopontin regulation, Clin. Diagn. Lab. Immunol. 12 (2005) 206-212.

[24] G. Schulz, A.C. Renkl, A. Seier, L. Liaw, J.M. Weiss, Regulated osteopontin expression by dendritic cells decisively affects their migratory capacity, J. Invest. Dermatol. 128 (2008) 2541-2544.

[25] A.C. Renkl, J. Wussler, T. Ahrens, K. Thoma, S. Kon, T. Uede, et al., Osteopontin functionally activates dendritic cells and induces their differentiation toward a Th1-polarizing phenotype, Blood 106 (2005) 946-955.

[26] J. Sodek, B. Ganss, McKee MD, Osteopontin. Crit Rev Oral Biol Med 11 (2000) 279-303.

[27] B. Kaleta, Role of osteopontin in systemic lupus erythematosus, Arch Immunol Ther Exp (Warsz) 62 (2014) 475-482.

[28] M.A. Briones-Orta, S.E. Avendano-Vazquez, D.I. Aparicio-Bautista, J.D. Coombes, G.F. Weber, W.K. Syn, Osteopontin splice variants and polymorphisms in cancer progression and prognosis, Biochim. Biophys. Acta Rev. Cancer 1868 (2017) 93-108. A.

[29] R. Wei, J.P.C. Wong, H.F. Kwok, Osteopontin - a promising biomarker for cancer therapy, J. Cancer 8 (2017) 2173-2183.

[30] E.R. Gimba, T.M. Tilli, Human osteopontin splicing isoforms: known roles, potential clinical applications and activated signaling pathways, Cancer Lett. 331 (2013) 11-17.

[31] J. Grassinger, D.N. Haylock, M.J. Storan, G.O. Haines, B. Williams, G.A. Whitty, et al., Thrombin-cleaved osteopontin regulates hemopoietic stem and progenitor cell functions through interactions with alpha9beta1 and alpha4beta1 integrins, Blood 114 (2009) 49-59.

[32] G.F. Weber, S. Ashkar, M.J. Glimcher, H. Cantor, Receptor-ligand interaction between CD44 and osteopontin (Eta-1), Science 271 (1996) 509-512.

[33] L. Shao, B. Zhang, L. Wang, L. Wu, Q. Kan, K. Fan, MMP-9-cleaved osteopontin isoform mediates tumor immune escape by inducing expansion of myeloid-derived suppressor cells, Biochem. Biophys. Res. Commun. 493 (2017) 1478-1484.

[34] S. Kon, Y. Nakayama, N. Matsumoto, K. Ito, M. Kanayama, C. Kimura, et al., A novel cryptic binding motif, LRSKSRSFQVSDEQY, in the C-terminal fragment of MMP-3/7-cleaved osteopontin as a novel ligand for alpha9beta1 integrin is involved in the anti-type II collagen antibody-induced arthritis, PLoS One 9 (2014) e116210.

[35] J.M. Weiss, A.C. Renkl, C.S. Maier, M. Kimmig, L. Liaw, T. Ahrens, et al., Osteopontin is involved in the initiation of cutaneous contact hypersensitivity by inducing Langerhans and dendritic cell migration to lymph nodes, J. Exp. Med. 194 (2001) 1219-1229.

[36] Z. Shao, J. Morser, L.L. Leung, Thrombin cleavage of osteopontin disrupts a prochemotactic sequence for dendritic cells, which is compensated by the release of its pro-chemotactic C-terminal fragment, J. Biol. Chem. 289 (2014) 27146-27158.

[37] T. Alissafi, E. Kourepini, D.C.M. Simoes, N. Paschalidis, M. Aggelakopoulou, T. Sparwasser, et al., Osteopontin promotes protective antigenic tolerance against experimental allergic airway disease, J. Immunol. 200 (2018) 1270-1282.

[38] D.T. Denhardt, M. Noda, A.W. O'Regan, D. Pavlin, J.S. Berman, Osteopontin as a means to cope with environmental insults: regulation of inflammation, tissue remodeling, and cell survival, J. Clin. Invest. 107 (2001) 1055-1061.

[39] J.W. Leavenworth, B. Verbinnen, J. Yin, H. Huang, H. Cantor, A p85alpha-osteopontin axis couples the receptor ICOS to sustained Bcl-6 expression by follicular helper and regulatory T cells, Nat. Immunol. 16 (2015) 96-106.

[40] J.W. Leavenworth, B. Verbinnen, Q. Wang, E. Shen, H. Cantor, Intracellular osteopontin regulates homeostasis and function of natural killer cells, Proc Natl Acad Sci U S A 112 (2015) 494-499.

[41] M. Inoue, Y. Moriwaki, T. Arikawa, Y.H. Chen, Y.J. Oh, T. Oliver, et al., Cutting edge: critical role of intracellular osteopontin in antifungal innate immune responses, J. Immunol. 186 (2011) 19-23.

[42] K. Zhao, M. Zhang, L. Zhang, P. Wang, G. Song, B. Liu, et al., Intracellular osteopontin stabilizes TRAF3 to positively regulate innate antiviral response, Sci. Rep. 6 (2016) 23771.

[43] M. Kanayama, S. Xu, K. Danzaki, J.R. Gibson, M. Inoue, S.G. Gregory, et al., Skewing of the population balance of lymphoid and myeloid cells by secreted and intracellular osteopontin, Nat. Immunol. 18 (2017) 973-984.

[44] A. Junaid, M.C. Moon, G.E. Harding, P. Zahradka, Osteopontin localizes to the nucleus of 293 cells and associates with polo-like kinase-1, Am. J. Physiol., Cell Physiol. 292 (2007) C919-26.

[45] L.F. Kraus, N. Scheurmann, D.F. Frenzel, A. Tasdogan, J.M. Weiss, 9-cis-Retinoic acid induces a distinct regulatory dendritic cell phenotype that modulates murine delayed-type allergy, Contact Derm. 78 (2018) 41-54.

[46] F. Blengio, F. Raggi, D. Pierobon, P. Cappello, A. Eva, M. Giovarelli, et al., The hypoxic environment reprograms the cytokine/chemokine expression profile of human mature dendritic cells, Immunobiology 218 (2013) 76-89.

[47] M. Yang, C. Ma, S. Liu, J. Sun, Q. Shao, W. Gao, et al., Hypoxia skews dendritic cells to a T helper type 2-stimulating phenotype and promotes tumour cell migration by dendritic cell-derived osteopontin, Immunology 128 (2009) e237-49.

[48] V. Salvi, S. Scutera, S. Rossi, M. Zucca, M. Alessandria, D. Greco, et al., Dual regulation of osteopontin production by TLR stimulation in dendritic cells, J. Leukoc. Biol. 94 (2013) 147-158.

[49] M.L. Shinohara, L. Lu, J. Bu, M.B. Werneck, K.S. Kobayashi, L.H. Glimcher, et al., Osteopontin expression is essential for interferon-alpha production by plasmacytoid dendritic cells, Nat. Immunol. 7 (2006) 498-506.

[50] M.L. Shinohara, J.H. Kim, V.A. Garcia, H. Cantor, Engagement of the type I interferon receptor on dendritic cells inhibits T helper 17 cell development: role of intracellular osteopontin, Immunity 29 (2008) 68-78. 
[51] G. Murugaiyan, A. Mittal, H.L. Weiner, Identification of an IL-27/osteopontin axis in dendritic cells and its modulation by IFN-gamma limits IL-17-mediated autoimmune inflammation, Proc. Natl. Acad. Sci. U S A 107 (2010) 11495-11500.

[52] S. Konno, J.A. Eckman, B. Plunkett, X. Li, J.S. Berman, J. Schroeder, et al., Interleukin-10 and Th2 cytokines differentially regulate osteopontin expression in human monocytes and dendritic cells, J. Interferon Cytokine Res. 26 (2006) $562-567$.

[53] M. Hulsmans, H.B. Sager, J.D. Roh, M. Valero-Munoz, N.E. Houstis, Y. Iwamoto, et al., Cardiac macrophages promote diastolic dysfunction, J. Exp. Med. 215 (2018) 423-440.

[54] S. De Keijzer, M.B. Meddens, R. Torensma, A. Cambi, The multiple faces of prostaglandin E2 G-protein coupled receptor signaling during the dendritic cell life cycle, Int. J. Mol. Sci. 14 (2013) 6542-6555.

[55] P. Kalinski, Regulation of immune responses by prostaglandin E2, J. Immunol. 188 (2012) 21-28.

[56] S. Scutera, V. Salvi, L. Lorenzi, G. Piersigilli, S. Lonardi, D. Alotto, et al., Adaptive regulation of osteopontin production by dendritic cells through the bidirectional interaction with mesenchymal stromal cells, Front. Immunol. 9 (2018) 1207.

[57] S. Sozzani, Dendritic cell trafficking: more than just chemokines, Cytokine Growth Factor Rev, 16 (2005) 581-592.

[58] L. Tiberio, A. Del Prete, T. Schioppa, F. Sozio, D. Bosisio, S. Sozzani, Chemokine and chemotactic signals in dendritic cell migration, Cell. Mol. Immunol. 15 (2018) 346-352.

[59] M.D. Begum, M. Umemura, S. Kon, A. Yahagi, S. Hamada, K. Oshiro, et al, Suppression of the bacterial antigen-specific T cell response and the dendritic cell migration to the lymph nodes by osteopontin, Microbiol. Immunol. 51 (2007) 135-147.

[60] S. Sozzani, P. Allavena, G. D’Amico, W. Luini, G. Bianchi, M. Kataura, et al., Differential regulation of chemokine receptors during dendritic cell maturation: a model for their trafficking properties, J. Immunol, 161 (1998) 1083-1086.

[61] M.L. Shinohara, H. Cantor, The bridge between dendritic cells and asthma, Nat. Med. 13 (2007) 536-538.

[62] H.J. de Heer, H. Hammad, T. Soullie, D. Hijdra, N. Vos, M.A. Willart, et al., Essential role of lung plasmacytoid dendritic cells in preventing asthmatic reactions to harmless inhaled antigen, J. Exp. Med. 200 (2004) 89-98.

[63] B.N. Lambrecht, M. De Veerman, A.J. Coyle, J.C. Gutierrez-Ramos, K. Thielemans, R.A. Pauwels, Myeloid dendritic cells induce Th2 responses to inhaled antigen, leading to eosinophilic airway inflammation, J. Clin. Invest. 106 (2000) 551-559.

[64] M.J. van Helden, B.N. Lambrecht, Dendritic cells in asthma, Curr. Opin. Immunol. 25 (2013) 745-754.

[65] G. Xanthou, T. Alissafi, M. Semitekolou, D.C. Simoes, E. Economidou, M. Gaga, et al., Osteopontin has a crucial role in allergic airway disease through regulation of dendritic cell subsets, Nat. Med. 13 (2007) 570-578.

[66] D.F. Frenzel, J.M. Weiss, Osteopontin and allergic disease: pathophysiology and implications for diagnostics and therapy, Expert Rev. Clin. Immunol. 7 (2011) 93-109.

[67] T. Sato, T. Nakai, N. Tamura, S. Okamoto, K. Matsuoka, A. Sakuraba, et al., Osteopontin/Eta-1 upregulated in Crohn's disease regulates the Th1 immune response, Gut 54 (2005) 1254-1262

[68] A.M. Seier, A.C. Renkl, G. Schulz, T. Uebele, A. Sindrilaru, S. Iben, et al., Antigenspecific induction of osteopontin contributes to the chronification of allergic contact dermatitis, Am. J. Pathol. 176 (2010) 246-258.

[69] M. Kurokawa, S. Konno, A. Takahashi, B. Plunkett, S.R. Rittling, Y. Matsui, et al., Regulatory role of DC-derived osteopontin in systemic allergen sensitization, Eur. J. Immunol. 39 (2009) 3323-3330.

[70] M. Larche, C.A. Akdis, R. Valenta, Immunological mechanisms of allergen-specific immunotherapy, Nat. Rev. Immunol. 6 (2006) 761-771.

[71] G. Cui, J. Chen, J. He, C. Lu, Y. Wei, L. Wang, et al., Osteopontin promotes dendritic cell maturation and function in response to HBV antigens, Drug Des. Devel. Ther. 9 (2015) 3003-3016.

[72] M. Cella, F. Facchetti, A. Lanzavecchia, M. Colonna, Plasmacytoid dendritic cells activated by influenza virus and CD40L drive a potent TH1 polarization, Nat. Immunol. 1 (2000) 305-310.

[73] A. Krug, S. Rothenfusser, V. Hornung, B. Jahrsdorfer, S. Blackwell, Z.K. Ballas, et al., Identification of $\mathrm{CpG}$ oligonucleotide sequences with high induction of IFNalpha/beta in plasmacytoid dendritic cells, Eur. J. Immunol. 31 (2001) 2154-2163.

[74] S. Konno, M. Kurokawa, T. Uede, M. Nishimura, S.K. Huang, Role of osteopontin, a multifunctional protein, in allergy and asthma, Clin. Exp. Allergy 41 (2011) 1360-1366.

[75] E. Uchio, N. Matsuura, K. Kadonosono, S. Ohno, T. Uede, Tear osteopontin levels in patients with allergic conjunctival diseases, Graefes Arch. Clin. Exp. Ophthalmol. 240 (2002) 924-928.

[76] L.L. Shi, J. Song, P. Xiong, P.P. Cao, B. Liao, J. Ma, et al., Disease-specific T-helper cell polarizing function of lesional dendritic cells in different types of chronic rhinosinusitis with nasal polyps, Am. J. Respir. Crit. Care Med. 190 (2014) 628-638.

[77] J. Deckers, K. De Bosscher, B.N. Lambrecht, H. Hammad, Interplay between bar rier epithelial cells and dendritic cells in allergic sensitization through the lung and the skin, Immunol. Rev. 278 (2017) 131-144.

[78] B. Kim, T.H. Kim, Fundamental role of dendritic cells in inducing Th2 responses, Korean J. Intern. Med. 33 (2018) 483-489.

[79] A. Nagasaka, H. Matsue, H. Matsushima, R. Aoki, Y. Nakamura, N. Kambe, et al., Osteopontin is produced by mast cells and affects IgE-mediated degranulation and migration of mast cells, Eur. J. Immunol. 38 (2008) 489-499.

[80] D.D. Patel, V.K. Kuchroo, Th17 cell pathway in human immunity: lessons from genetics and therapeutic interventions, Immunity 43 (2015) 1040-1051.

[81] A. Awasthi, Y. Carrier, J.P. Peron, E. Bettelli, M. Kamanaka, R.A. Flavell, et al., A dominant function for interleukin 27 in generating interleukin 10-producing antiinflammatory T cells, Nat. Immunol. 8 (2007) 1380-1389.

[82] M. Teixeira-Coelho, A. Cruz, J. Carmona, C. Sousa, D. Ramos-Pereira, A.L. Saraiva, et al., TLR2 deficiency by compromising p19 (IL-23) expression limits Th 17 cell responses to Mycobacterium tuberculosis, Int. Immunol. 23 (2011) 89-96.

[83] M.R. Kim, S.W. Hong, E.B. Choi, W.H. Lee, Y.S. Kim, S.G. Jeon, et al., Staphylococcus aureus-derived extracellular vesicles induce neutrophilic pulmonary inflammation via both Th1 and Th17 cell responses, Allergy 67 (2012) $1271-1281$.

[84] E. Hoe, J. Anderson, J. Nathanielsz, Z.Q. Toh, R. Marimla, A. Balloch, et al., The contrasting roles of Th17 immunity in human health and disease, Microbiol. Immunol. 61 (2017) 49-56.

[85] G. Murugaiyan, A. Mittal, H.L. Weiner, Increased osteopontin expression in dendritic cells amplifies IL-17 production by CD4 + T cells in experimental autoimmune encephalomyelitis and in multiple sclerosis, J. Immunol. 181 (2008) $7480-7488$

[86] F. Buback, A.C. Renkl, G. Schulz, J.M. Weiss, Osteopontin and the skin: multiple emerging roles in cutaneous biology and pathology, Exp. Dermatol. 18 (2009) 750-759.

[87] E. Glitzner, A. Korosec, P.M. Brunner, B. Drobits, N. Amberg, H.B. Schonthaler, et al., Specific roles for dendritic cell subsets during initiation and progression of psoriasis, EMBO Mol. Med. 6 (2014) 1312-1327.

[88] C. Wohn, J.L. Ober-Blobaum, S. Haak, S. Pantelyushin, C. Cheong, S.P. Zahner et al., Langerin(neg) conventional dendritic cells produce IL-23 to drive psoriatic plaque formation in mice, Proc Natl Acad Sci U S A 110 (2013) 10723-10728.

[89] C. Albanesi, C. Scarponi, D. Bosisio, S. Sozzani, G. Girolomoni, Immune function and recruitment of plasmacytoid dendritic cells in psoriasis, Autoimmunity 43 (2010) 215-219.

[90] Y.J. Chen, J.L. Shen, C.Y. Wu, Y.T. Chang, C.M. Chen, F.Y. Lee, Elevated plasma osteopontin level is associated with occurrence of psoriasis and is an unfavorable cardiovascular risk factor in patients with psoriasis, J. Am. Acad. Dermatol. 60 (2009) 225-230.

[91] S. Keely, N.J. Talley, P.M. Hansbro, Pulmonary-intestinal cross-talk in mucosal inflammatory disease, Mucosal Immunol. 5 (2012) 7-18.

[92] E. Kourepini, M. Aggelakopoulou, T. Alissafi, N. Paschalidis, D.C. Simoes, V. Panoutsakopoulou, Osteopontin expression by CD103- dendritic cells drives intestinal inflammation, Proc Natl Acad Sci U S A 111 (2014) E856-65.

[93] M. Shan, X. Yuan, L.Z. Song, L. Roberts, N. Zarinkamar, A. Seryshev, et al., Cigarette smoke induction of osteopontin (SPP1) mediates T(H)17 inflammation in human and experimental emphysema, Sci. Transl. Med. 4 (2012) 117ra9.

[94] T. Ruzicka, C.W. Lynde, G.B. Jemec, T. Diepgen, J. Berth-Jones, P.J. Coenraads, et al., Efficacy and safety of oral alitretinoin (9-cis retinoic acid) in patients with severe chronic hand eczema refractory to topical corticosteroids: results of a randomized, double-blind, placebo-controlled, multicentre trial, Br. J. Dermatol. 158 (2008) 808-817.

[95] C.L. Scott, A.M. Aumeunier, A.M. Mowat, Intestinal CD103 + dendritic cells: master regulators of tolerance? Trends Immunol. 32 (2011) 412-419.

[96] C.M. Sun, J.A. Hall, R.B. Blank, N. Bouladoux, M. Oukka, J.R. Mora, et al., Small intestine lamina propria dendritic cells promote de novo generation of Foxp3 T reg cells via retinoic acid, J. Exp. Med. 204 (2007) 1775-1785.

[97] L.A. Shevde, R.S. Samant, Role of osteopontin in the pathophysiology of cancer, Matrix Biol. 37 (2014) 131-141.

[98] M.A. Icer, M. Gezmen-Karadag, The multiple functions and mechanisms of osteopontin, Clin. Biochem. 59 (2018) 17-24.

[99] H. Zhao, Q. Chen, A. Alam, J. Cui, K.C. Suen, A.P. Soo, et al., The role of osteopontin in the progression of solid organ tumour, Cell Death Dis. 9 (2018) 356.

[100] R.M. Awad, Y. De Vlaeminck, J. Maebe, C. Goyvaerts, K. Breckpot, Turn back the TIMe: targeting tumor infiltrating myeloid cells to revert Cancer progression, Front. Immunol. 9 (2018) 1977.

[101] E.J. Clappaert, A. Murgaski, H. Van Damme, M. Kiss, D. Laoui, Diamonds in the rough: harnessing tumor-associated myeloid cells for Cancer therapy, Front. Immunol. 9 (2018) 2250.

[102] L. Dolcetti, E. Peranzoni, V. Bronte, Measurement of myeloid cell immune sup pressive activity, Curr. Protoc. Immunol. 91 (1) (2010) 14.17.1-14.17.25 Chapter 14:Unit 147

[103] G. Rao, L. Du, Q. Chen, Osteopontin, a possible modulator of cancer stem cells and their malignant niche, Oncoimmunology 2 (2013) e24169.

[104] C. Chiodoni, S. Sangaletti, M.P. Colombo, Matricellular proteins tune myeloidderived suppressor cell recruitment and function in breast cancer, J. Leukoc. Biol. 102 (2017) 287-292.

[105] E.K. Kim, I. Jeon, H. Seo, Y.J. Park, B. Song, K.A. Lee, et al., Tumor-derived osteopontin suppresses antitumor immunity by promoting extramedullary myelopoiesis, Cancer Res. 74 (2014) 6705-6716.

[106] S. Sangaletti, C. Tripodo, S. Sandri, I. Torselli, C. Vitali, C. Ratti, et al., Osteopontin shapes immunosuppression in the metastatic niche, Cancer Res. 74 (2014) $4706-4719$.

[107] J.D. Klement, A.V. Paschall, P.S. Redd, M.L. Ibrahim, C. Lu, D. Yang, et al., An osteopontin/CD44 immune checkpoint controls CD8 + T cell activation and tumo immune evasion, J. Clin. Invest. 128 (2018) 5549-5560.

[108] F. Veglia, D.I. Gabrilovich, Dendritic cells in cancer: the role revisited, Curr. Opin. Immunol. 45 (2017) 43-51.

[109] J.R. Conejo-Garcia, M.R. Rutkowski, J.R. Cubillos-Ruiz, State-of-the-art of regulatory dendritic cells in cancer, Pharmacol. Ther. 164 (2016) 97-104.

[110] A. Mancino, T. Schioppa, P. Larghi, F. Pasqualini, M. Nebuloni, I.H. Chen, et al., 
Divergent effects of hypoxia on dendritic cell functions, Blood 112 (2008) 3723-3734.

[111] C. Nombela-Arrieta, J. Ritz, L.E. Silberstein, The elusive nature and function of mesenchymal stem cells, Nat. Rev. Mol. Cell Biol. 12 (2011) 126-131.

[112] M. Wang, Q. Yuan, L. Xie, Mesenchymal stem cell-based immunomodulation: properties and clinical application, Stem Cells Int. 2018 (2018) 3057624.

[113] A. Sapoznikov, Y. Pewzner-Jung, V. Kalchenko, R. Krauthgamer, I. Shachar, S. Jung, Perivascular clusters of dendritic cells provide critical survival signals to B cells in bone marrow niches, Nat. Immunol. 9 (2008) 388-395.

[114] J. Dokic, S. Tomic, M. Markovic, P. Milosavljevic, M. Colic, Mesenchymal stem cells from periapical lesions modulate differentiation and functional properties of monocyte-derived dendritic cells, Eur. J. Immunol. 43 (2013) 1862-1872.

[115] Q. Chen, P. Shou, C. Zheng, M. Jiang, G. Cao, Q. Yang, et al., Fate decision of mesenchymal stem cells: adipocytes or osteoblasts? Cell Death Differ. 23 (2016) 1128-1139.

[116] A.M. Silva, M.I. Almeida, J.H. Teixeira, A.F. Maia, G.A. Calin, M.A. Barbosa, et al., Dendritic cell-derived extracellular vesicles mediate mesenchymal Stem/Stromal cell recruitment, Sci. Rep. 7 (2017) 1667.

[117] W. Wang, P. Li, W. Li, J. Jiang, Y. Cui, S. Li, et al., Osteopontin activates mesenchymal stem cells to repair skin wound, PLoS One 12 (2017) e0185346.

[118] Y. Wang, X. Chen, W. Cao, Y. Shi, Plasticity of mesenchymal stem cells in immunomodulation: pathological and therapeutic implications, Nat. Immunol. 15 (2014) 1009-1016.

[119] L. de Girolamo, E. Lucarelli, G. Alessandri, M.A. Avanzini, M.E. Bernardo, E. Biagi, et al., Mesenchymal stem/stromal cells: a new' 'cells as drugs" paradigm. Efficacy and critical aspects in cell therapy, Curr. Pharm. Des. 19 (2013) 2459-2473.

[120] K.C. Rustad, G.C. Gurtner, Mesenchymal stem cells home to sites of injury and inflammation, Adv. Wound Care (New Rochelle) 1 (2012) 147-152.

[121] G.M. Spaggiari, L. Moretta, Cellular and molecular interactions of mesenchyma stem cells in innate immunity, Immunol. Cell Biol. 91 (2013) 27-31.

[122] R. Torres, A. Herrerias, M. Serra-Pages, A. Marco, J. Plaza, C. Costa-Farre, et al., Locally administered prostaglandin E2 prevents aeroallergen-induced airway sensitization in mice through immunomodulatory mechanisms, Pharmacol. Res. 70 (2013) 50-59.

[123] S.G. Royce, S. Rele, B.R.S. Broughton, K. Kelly, C.S. Samuel, Intranasal administration of mesenchymoangioblast-derived mesenchymal stem cells abrogates airway fibrosis and airway hyperresponsiveness associated with chronic allergic airways disease, Faseb J. 31 (2017) 4168-4178.

[124] M.J. Boumans, J.G. Houbiers, P. Verschueren, H. Ishikura, R. Westhovens, E. Brouwer, et al., Safety, tolerability, pharmacokinetics, pharmacodynamics and efficacy of the monoclonal antibody ASK8007 blocking osteopontin in patients with rheumatoid arthritis: a randomised, placebo controlled, proof-of-concept study, Ann. Rheum. Dis. 71 (2012) 180-185.

[125] C.E. West, A.S. Kvistgaard, J.M. Peerson, S.M. Donovan, Y.M. Peng, B. Lonnerdal, Effects of osteopontin-enriched formula on lymphocyte subsets in the first 6 months of life: a randomized controlled trial, Pediatr. Res. 82 (2017) 63-71.

[126] N. Clemente, C. Comi, D. Raineri, G. Cappellano, D. Vecchio, E. Orilieri, et al., Role of anti-osteopontin antibodies in multiple sclerosis and experimental autoimmune encephalomyelitis, Front. Immunol. 8 (2017) 321

[127] B. Kaleta, The role of osteopontin in kidney diseases, Inflamm. Res. 68 (2019) 93-102.

[128] L.T. Wang, C.H. Ting, M.L. Yen, K.J. Liu, H.K. Sytwu, K.K. Wu, et al., Human mesenchymal stem cells (MSCs) for treatment towards immune- and inflammation-mediated diseases: review of current clinical trials, J. Biomed. Sci. 23 (2016) 76.

[129] M.E. Bernardo, W.E. Fibbe, Safety and efficacy of mesenchymal stromal cell therapy in autoimmune disorders, Ann. N. Y. Acad. Sci. 1266 (2012) 107-117.

[130] K. Chen, Q. Liu, L.L. Tsang, Q. Ye, H.C. Chan, Y. Sun, et al., Human MSCs promotes colorectal cancer epithelial-mesenchymal transition and progression via CCL5/ beta-catenin/Slug pathway, Cell Death Dis. 8 (2017) e2819.

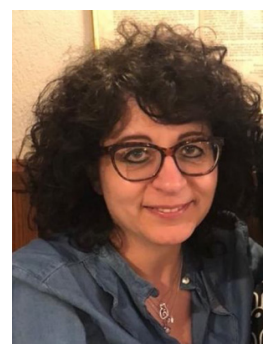

Annalisa Del Prete is Associate Professor in Clinical Pathology at the Department of Molecular and Translational Medicine of the University of Brescia, Italy.She received MD degree in 1995, and specialized in Clinical Biochemistry in 1999, at the University of Bari, Italy. She obtained her PhD at the "Mario Negri" Institute in Milan, where she started her research work focusing on the mechanisms regulating the migration of dendritic cells in in vitro and in vivo experimental models. The recent interests concern the study of the role of atypical chemokine receptors in the recruitment of leukocyte in the inflammatory sites and in tumor microenvironment.

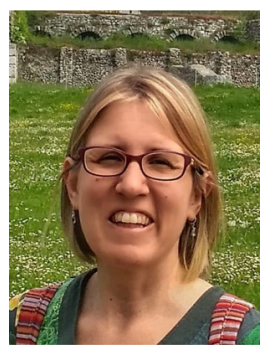

Sara Scutera is Assistant Professor at the Department of Public Health and Pediatric Sciences, University of Torino, Italy. She obtained her PhD in Microbiology and Virology at the Medical Faculty of the Torino University in 2006 and the $\mathrm{PhD}$ in Molecular Medicine, Immunology and Cellular Biology Specialization, at the University of Torino in 2012 Her research is focused on the antimicrobial mechanisms of dendritic cells and macrophages and in the processes in volved in their activation. She is also involved in the study of chronic inflammatory diseases and scar healing.

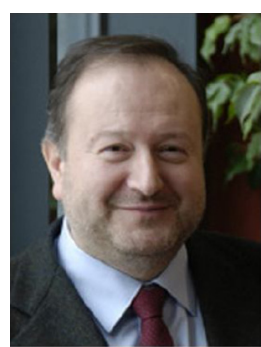

Silvano Sozzani is Professor in General Pathology and Chairman of the Department of Molecular and Translationa Medicine at the University of Brescia, Italy. In1983, he graduated from the school of Pharmacy of theUniversity of Pavia, Italy. In 1988, during his postdoctoralstage at the Department of Biochemistry atWake Forest University, NC, USA, he started to work onthe signal transduction of che motactic receptors. Sincethen his research interests have been focusing on therole chemokines and chemokine receptors in phagocyteand dendritic cell biology. These studies led to thedescription of the "chemokine receptor switch" duringdendritic cell maturation and to the characterization of new chemokines and chemokine receptors. $\mathrm{He}$ is member of the Editorial board ofseveral scientific journals.

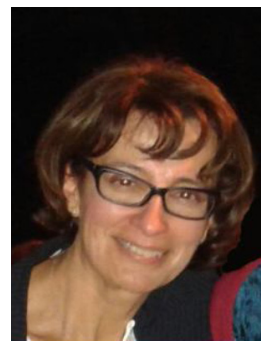

Tiziana Musso obtained her PhD in Biology in 1986 at the University of Torino. From 1988 to 1995 she was a Researcher at the Biological Carcinogenesis and Developmental Program, National Cancer Institute, Frederick,USA and she mainly studied the IL-2 signaling pathway in human monocytes. She is Associate Professor at the School of Medicine, University of Torino. Her research is focused on the impact of the immune and inflammatory response in microbial infections with emphasis on theregulation of cytokines in dendritic cells. 